\title{
Variation of sulfur isotopes in sediments from the late Quaternary Qiantang River incised valley, Eastern China
}

\author{
K.X. JIANG ${ }^{1}$, C.M. LIN ${ }^{1}$ * , X. ZHANG ${ }^{1}$
}

${ }^{1}$ State Key Laboratory for Mineral Deposits Research, School of Earth Sciences and Engineering, Nanjing University, 163 Xianlin Road, Nanjing 210023, China (*correspondence: cmlin@nju.edu.cn)

The sediments of the late Quaternary Qiantang River incised valley have experienced dramatic evolution such as sea level and climate changed. The inorganic and organic geochemistry features of sediment samples were analyzed in detail, which obtained from the SE3 core drilled in the late Quaternary Qiantang River incised valley. The sediments were divided into five sedimentary units, Units I IV, in ascending order, based on their lithological features, and variations in the vertical distribution of geochemical parameters, such as TOC, TS, trace elements, biomarkers, stable isotopes of oxygen, carbon, and sulfur. Based on the multiple proxies, we inferred that Unit I was floodplain facies deposited in fresh water, Unit II was paleo-estuary facies, Unit III may be storm deposits (the maxium values of $\delta^{13} \mathrm{C}_{\text {carbonate }}$ and $\delta^{18} \mathrm{O}_{\text {carbonate }}$ were recorded), Unit IV and $\mathrm{V}$ both were nearshore facies. While Unit V was shallower than Unit IV.

Unit I sediments exhibit median (range) of $\delta^{34} \mathrm{~S}_{\mathrm{AVS}}$ and $\delta^{34} \mathrm{~S}_{\mathrm{py}}$ values of $10.35 \%$ (8.11\% $~ 12.37 \%$ o $)$ and $7.84 \% 0$ (1.61\%o 12.03\%o), respectively, yielding TOC/Spy ratios of 178.89 (45.76 378.39). Unit II sediments exhibit median (range) of $\delta^{34} \mathrm{~S}_{\mathrm{AVS}}$ and $\delta^{34} \mathrm{~S}_{\mathrm{py}}$ values of $16.79 \%$ $(14.58 \% \sim 20.57 \%$ o $)$ and $13.11 \%$ o $\quad(9.29 \%$ \% $15.42 \%$ ), respectively, yielding TOC/Spy ratios of 8.97 (0.70 17.00). Unit III sediments exhibit median (range) of $\delta^{34} \mathrm{~S}_{\mathrm{Avs}}$ and $\delta^{34} S_{\text {py }}$ values of $14.68 \%$ o $(0.98 \% \sim 35.00 \%$ o $)$ and $-1.63 \%$ ($19.72 \%$ 15.76\%o), respectively, yielding TOC/Spy ratios of 0.65 (0.47 0.94). Unit IV sediments exhibit median (range) of $\delta^{34} \mathrm{~S}_{\mathrm{AVS}}$ and $\delta^{34} \mathrm{~S}_{\mathrm{py}}$ values of $3.43 \%$ (-2.95\% $11.76 \%$ ) and $-6.62 \%$ o (-15.80\% $1.96 \%$ ), respectively, yielding TOC/Spy ratios of $2.38(1.31 \sim 3.33)$. Unit $\mathrm{V}$ sediments exhibit median (range) of $\delta^{34} \mathrm{~S}_{\mathrm{AvS}}$ and $\delta^{34} \mathrm{~S}_{\mathrm{py}}$ values of $17.77 \%$ $(5.46 \%$ o $50.77 \%$ o) and $8.78 \%$ o $\quad(-2.87 \%$ $45.30 \%$ ), respectively, yielding TOC/Spy ratios of 5.64 (1.72 9.72). Compare to other Units, the $\delta^{34} S_{A v s}$ and $\delta^{34} S_{p y}$ values of Unit III fluctuate sharply, which was probably caused by the rapid deposition of storm surge and strong mixing of fresh water and sea water. 